\title{
Kinematics Analysis and Simulation of a Rocker- Bogie Mobile Robot
}

\author{
Mohammad Reza Elhami ${ }^{1}$, Iman Dashti ${ }^{2}$
}

\begin{abstract}
For a wheeled mobile robot in rough terrain, one of the known suspension systems is the rocker-bogie mechanism. The high mobility of the robot, moving in 3 dimensions with 6 degrees of freedom makes the kinematics modelling as a challenging task. In this paper, a full 6-DOF kinematic model of a rocker-bogie mobile robot is presented. The A matrices has been derived based on Denavit-Hartenberg coordinate transformation approach. The kinematic equations and Jacobian matrices for the wheels are derived which relates the rover velocity vector with wheel angular velocities and joint angular rates. Furthermore, a rover-terrain model is developed to obtain the necessary joint angles and some attitude angles by solving nonlinear optimization equations. Finally, the robot model is also constructed in MD Adams and simulations are carried out to verify the kinematics model. The results show very close match of kinematic model and simulation in rough terrain trajectory.
\end{abstract}

Keywords- Mobile robot, Rocker-Bogie, Kinematic analysis, Rough terrain

\section{Introduction}

Rescue operations and future space explorations will require high mobility robots to perform intricate tasks in challenging uneven terrain. Examples of rough terrain applications for such robotic systems can be found in the forestry, mining industries, and in hazardous material handling applications. The high mobility of these robots, moving in 3 dimensions with 6 degrees of freedom $(\mathrm{X}, \mathrm{Y}, \mathrm{Z}$, pitch, yaw, roll), makes the kinematics modelling a challenging task than the robots which move on flat and smooth surface (3 degrees of freedom : X, Y, rotation about $\mathrm{Z}$ axis).

So far a variety of approaches for kinematics modelling have been implemented which can be roughly divided into two methods-one related the geometric approach (Cox and Wilfong, 1990; Iagnemma et al., 1999) and the other concerning transformation approach. One of the earliest works on the formulation of wheeled mobile robot's kinematics equations of motion has been studied by Muir et al. (1987). In this work, matrix coordinate transformation algebra is developed to derive the equations of the motion of mobile robots. Due to the simplifying assumptions, this and similar approaches are only applicable to motion in 2dimensional space, i.e. translation in the $x-y$ plane and yaw rotation. A general approach to kinematic modelling of articulated rovers traversing uneven terrain was developed by Tarokh et al. (1999). Later on, three forms of kinematics,

${ }^{1}$ Mohammad Reza Elhami, Associate Prof.

${ }^{2}$ Iman Dashti, Graduted Student

Imam Hosein University

Tehran, Iran i.e. navigation, actuation and slip kinematics were identified and the equations and application of each were discussed. Kinematics analysis of six-wheeled rovers (such as the vehicle "Sojourner" of JPL) was studied by Chottiner (1992), Linderman and Eisen (1992), Hacot et al. (1998).

In this paper the kinematics modelling of a six-wheel rocker-bogie mobile robot based on above literature is deduced. The kinematics model will be useful and fundamental to subsequent studies on trajectory tracking and motion control of the mobile robot.

Fig. 1 shows the schematic diagram of a rocker bogie rover. The rover has different elements, which are assumed to be rigid. The suspension mechanism consists of several joints and by adjusting its joints the rover is capable of locomotion over various uneven terrains. The rocker bogie structure has six independently driven wheels which are mounted on an articulated passive suspension system. The suspension system consists of two rocker arms connected to the rover body. Each rocker has a rear wheel connected to one end and a bogie connected to the other end. The bogie is connected to the rocker with a free pivoting joint. At each end of the bogie there is a drive wheel. The rockers are connected to the rover body with a differential joint.

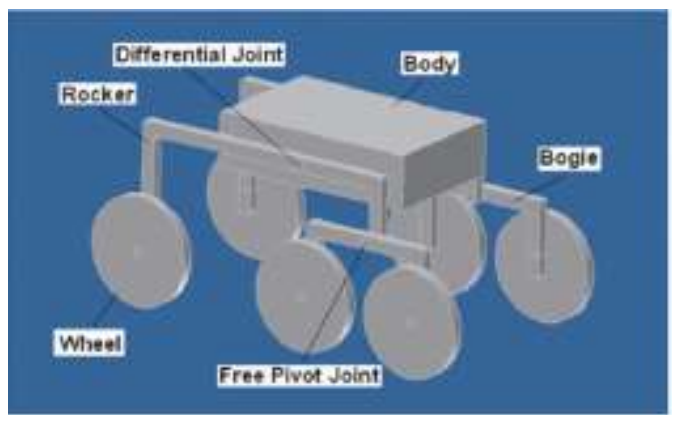

Figure 1. Example of a Rocker-Bogie System

\section{Kinematics Formulation}

\section{A. Coordinate Frames}

According to the D-H method (Craig, 1989), a coordinate frame is introduced on each joint. We define coordinate frames as in Fig. 2. The subscripts for the coordinate frames are as follows: $\mathrm{R}$ : robot reference frame, $\mathrm{D}$ : differential joint, $\mathrm{B}_{\mathrm{i}}$ : left and right bogies $(\mathrm{i}=1,2), \mathrm{S}_{\mathrm{i}}$ : steering coordinate frames $\quad(i=1-6)$ and $A_{i}$ : Axle of all wheels $(i=1-6)$. 


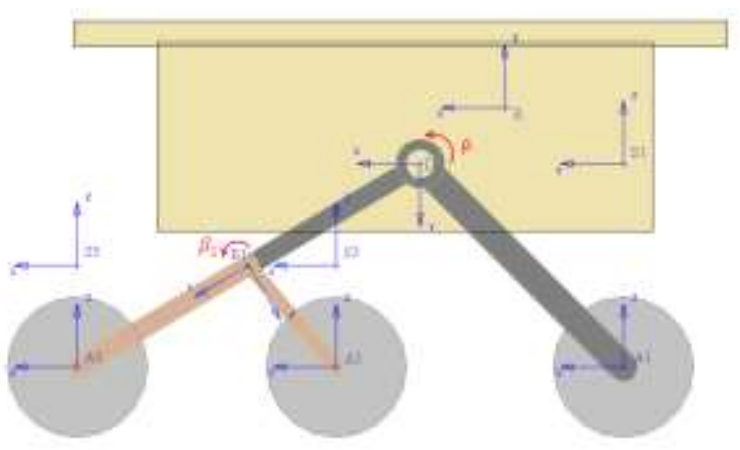

a) Side View

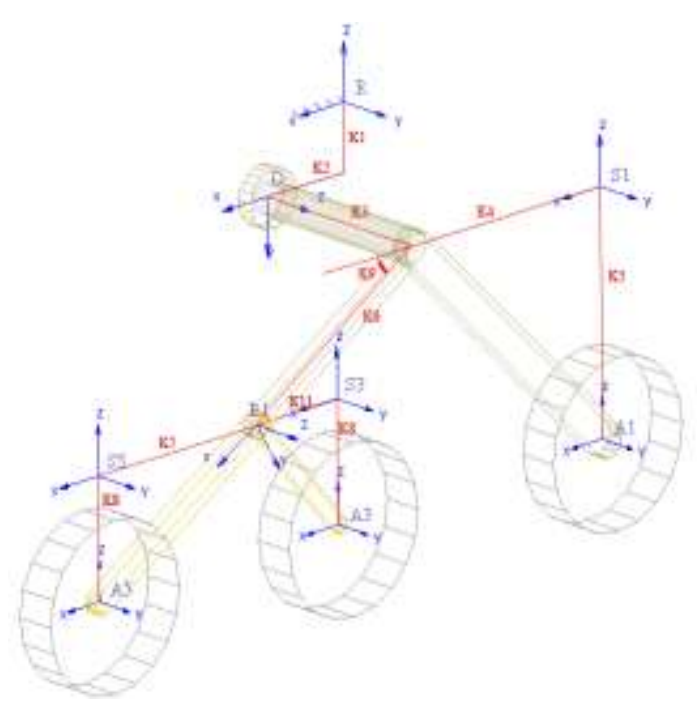

b) Perspective View

Figure 2. Coordinate Frames for Rover Left Side

The rover configuration vector $\mathrm{U}=\left[\mathrm{XYZ} \Phi_{\mathrm{x}} \Phi_{y} \Phi_{\mathrm{z}}\right]^{\mathrm{T}}$, is defined relative to the world coordinate frame $\mathrm{W}$, where $\left(\mathrm{X} \mathrm{Y} \mathrm{Z)} \mathrm{is} \mathrm{the} \mathrm{position} \mathrm{and}\left(\Phi_{x} \Phi_{y} \Phi_{z}\right)\right.$ is the orientation with roll, pitch and heading respectively. The Rover joint variable vector (q) consists of suspension joints angles: steering angles $\left(\Psi_{1}, \Psi_{2}\right)$ x rocker angle $(\rho)$, left and right bogie angles $\left(\beta_{1}, \beta_{2}\right)$.

A transformation matrix can be defined between two consequent frames, using the D-H parameters, $\mathrm{a}_{\mathrm{i}}, \mathrm{\alpha}_{\mathrm{i}}, \mathrm{d}_{\mathrm{i}}, \mathrm{\theta}_{\mathrm{i}}$.

$$
T_{i-1, i}=\left[\begin{array}{cccc}
c \theta_{i} & -s \theta_{i} c \alpha_{i} & s \theta_{i} s \alpha_{i} & a_{i} c \theta_{i} \\
s \theta_{i} & c \theta_{i} c \alpha_{i} & -c \theta_{i} s \alpha_{i} & a_{i} s \theta_{i} \\
0 & s \alpha_{i} & c \alpha_{i} & d_{i} \\
0 & 0 & 0 & 1
\end{array}\right]
$$

Where $\mathrm{c}(\alpha)=\cos (\alpha), \mathrm{s}(\alpha)=\sin (\alpha)$.
The transformations from the robot reference frame (R) to the wheel axle frames $\left(A_{i}\right)$ are obtained by cascading the individual transformations which gives the position of each wheel in rover reference frame. Then the transformation matrix for each wheel is obtained as:

$$
\begin{aligned}
& \text { (2) } T_{R_{s}, A_{\mathrm{i}}}=T_{R_{\mathbb{N}},} \cdot T_{D_{j} S_{\mathrm{i}}} \cdot T_{S_{\mathrm{i}}, A_{\mathrm{i}}} \quad \mathrm{i}=1,2 \\
& \text { (3) } T_{R_{2}, A_{1}}=T_{R_{1}, D^{x}} T_{D_{i} B_{2}} \cdot T_{B_{2}, S_{1}} T_{S_{1}, A_{4}} \quad i=3,5 \\
& \text { (4) } T_{R_{i}, A_{i}}=T_{R, D} \cdot T_{D_{i} B_{2}} \cdot T_{B_{2}, S_{i}} \cdot T_{S_{i,}, A_{i}} \quad i=4,6
\end{aligned}
$$

Table 1 shows $\mathrm{D}-\mathrm{H}$ parameters representing transformation between two consequent frames.

TABLE I. D-H PARAMETERS FOR COORDINATE FRAMES.

\begin{tabular}{|c|c|c|c|c|}
\hline Frame & $\boldsymbol{\theta}(\mathbf{d e g})$ & $\mathbf{d}(\mathbf{c m})$ & $\mathbf{a}(\mathbf{c m})$ & $\boldsymbol{\alpha}(\mathbf{d e g})$ \\
\hline $\mathrm{D}$ & 0 & $\mathrm{~K} 1$ & $\mathrm{~K} 2$ & -90 \\
\hline $\mathrm{B} 1$ & $\mathrm{~K} 9+\rho$ & $\mathrm{K} 3$ & $\mathrm{~K} 6$ & 0 \\
\hline $\mathrm{B} 2$ & $\mathrm{~K} 9-\rho$ & $-\mathrm{K} 3$ & $\mathrm{~K} 6$ & 0 \\
\hline $\mathrm{S} 1$ & $\rho$ & $\mathrm{K} 3$ & $-\mathrm{K} 4$ & 90 \\
\hline $\mathrm{S} 2$ & $-\rho$ & $-\mathrm{K} 3$ & $-\mathrm{K} 4$ & 90 \\
\hline $\mathrm{S} 3$ & $1-\mathrm{K} 9 \beta$ & 0 & $-\mathrm{K} 11$ & 90 \\
\hline $\mathrm{S} 4$ & $2-\mathrm{K} 9 \beta$ & 0 & $-\mathrm{K} 11$ & 90 \\
\hline $\mathrm{S} 5$ & $1-\mathrm{K} 9 \beta$ & 0 & $\mathrm{~K} 7$ & 90 \\
\hline S6 & $2-\mathrm{K} 9 \beta$ & 0 & $\mathrm{~K} 7$ & 90 \\
\hline A1 & $1 \psi$ & $-\mathrm{K} 5$ & 0 & 0 \\
\hline A2 & $2 \psi$ & $-\mathrm{K} 5$ & 0 & 0 \\
\hline A3 & 0 & $-\mathrm{K} 8$ & 0 & 0 \\
\hline A4 & 0 & $-\mathrm{K} 8$ & 0 & 0 \\
\hline A5 & 0 & $-\mathrm{K} 8$ & 0 & 0 \\
\hline A6 & 0 & $-\mathrm{K} 8$ & 0 & 0 \\
\hline
\end{tabular}

$K_{1}=K_{2}=0 ; K_{3}=27 ; K_{4}=29$

$K_{5}=29 ; K_{6}=28.46 ; K_{7}=24.5$

$K_{8}=14.5 ; K_{9}=30.7 ; K_{10}=10$

$K_{11}=12.5$ 
Proc. of the Intl. Conf. on Advances in Civil, Structural and Mechanical Engineering - ACSM 2015.

Copyright $($ Institute of Research Engineers and Doctors, USA .All rights reserved.

ISBN: 978-1-63248-039-2 doi: 10.15224/ 978-1-63248-039-2-19

\section{B. The Wheel and Terrain Contact Model}

Assuming a single contact point between wheels and the ground, for each wheel a coordinate frame is defined at contact point. The contact coordinate frame $\mathrm{C}_{\mathrm{i}}$ is obtained from the axle coordinate frame, $A_{\mathrm{i}^{x}}$ by rotating $\delta_{\mathrm{i}}$ about the axle, then translating by the wheel radius in the negative $\mathrm{Z}$ direction. The corresponding transformation matrix from the axle $A_{\mathrm{i}}$ to contact $C_{\mathrm{i}}$ is given by:

$$
T_{W, R}=\left[\begin{array}{cccc}
c \delta_{i} & 0 & s \delta_{i} & -r s \delta_{i} \\
0 & 1 & 0 & 0 \\
-s \delta_{i} & 0 & c \delta_{i} & -r c \delta_{i} \\
0 & 0 & 0 & 1
\end{array}\right]
$$

Where $\delta_{i}$ is contact angle of wheel $i$ and $r$ is radius of wheels. However, this transformation does not include rolling or slip, and thus does not reflect motion. In order to include motion, we consider the motion frames $\overline{\mathrm{C}}_{\mathrm{i}}(\mathrm{t})=\mathrm{C}_{\mathrm{i}}(\mathrm{t}-\Delta \mathrm{t})$ and $\mathrm{C}_{\mathrm{i}}(\mathrm{t})$ as shown in Fig. 4 .

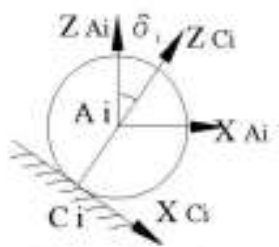

Figure 3. Wheel Contact Coordinate Frame

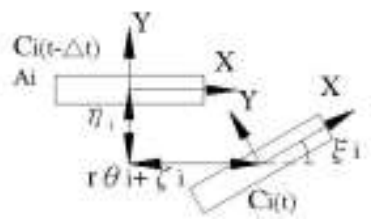

Figure 4. Wheel Motion Model

The transformation matrix for motion frames are derived using wheel rolling translation $\left(r \theta_{i}+\xi_{i}\right)$ along the $\mathrm{x}$-axis, where $\theta_{\mathrm{i}}$ is the angular rotation and $\xi_{\mathrm{i}}$ is the rolling slip, a wheel side slip translation $\left(\eta_{i}\right)$ along the y-axis, and a turn slip rotation $\left(E_{-i}\right)$ about the z-axis.

$$
\dot{T}_{\bar{C}, C}=\left[\begin{array}{cccc}
c \zeta_{i} & -s \zeta_{i} & 0 & r \theta+\xi_{i} \\
s \zeta_{i} & c \zeta_{i} & 0 & \eta_{i} \\
0 & 0 & 1 & 0 \\
0 & 0 & 0 & 1
\end{array}\right]
$$

\section{c. Wheel Jacobian Matrices}

In order to describe the relative motion of the rover body with terrain, similar to the instantaneous coincident coordinate frame $\overline{\mathrm{C}}_{\overline{\mathrm{i}}}$, we develop a rover body instantaneous coincident coordinate frame, $\overrightarrow{\mathrm{R}} . \mathrm{T}_{\mathrm{R}_{\mathrm{R}}}$ is an identity matrix and its derivative, $\mathrm{t}_{\mathbb{R}_{R}}$, has the following form:

$$
\dot{T}_{\bar{R}, R}=\left[\begin{array}{cccc}
0 & -\dot{\varphi}_{z} & \dot{\varphi}_{y} & \dot{x} \\
\dot{\varphi}_{z} & 0 & -\dot{\varphi}_{x} & \dot{y} \\
-\dot{\varphi}_{y} & \dot{\varphi}_{x} & 0 & \dot{z} \\
0 & 0 & 0 & 1
\end{array}\right]
$$

Defining $\mathrm{F}$ as the terrain coordinate frame, then figure 6 illustrates the relation of each part of the rover with the terrain.

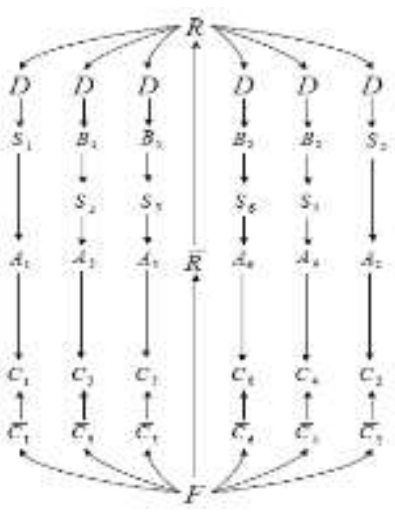

Figure 5. Transformation Loop

In view of the fact that the inverse of the transformation matrix is equal to the inverse chain transformation matrix, one has the following:

$$
\mathrm{T}_{\overline{\mathrm{R}}, \mathrm{R}}=\mathrm{T}_{\overline{\mathrm{R}}, \overline{\mathrm{C}}_{\mathrm{i}}} \cdot \mathrm{T}_{\overline{\mathrm{C}}_{\mathrm{j}}, \mathrm{R}}
$$

By evaluating the partial derivatives and substituting $\mathrm{t}_{\mathrm{R}_{\mathrm{R}}}$ in equation (7), velocity components of joint angles are factorized in the right side of equation (8) and velocity

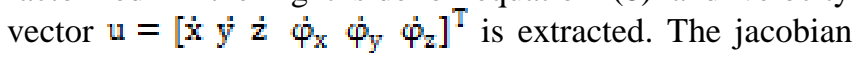
matrix for each wheel is derived as in equation (9):

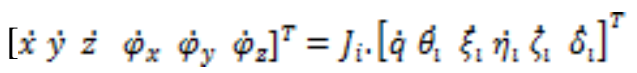

Where $\mathrm{q}$ is the joint variable vector defined in section 2.1 and its components is different for each wheel. Equation (9) describes the contribution of individual wheel motion and the connecting joints to the rover body motion. 


\section{D.Rover-Terrain Model}

The purpose of this subsection is to determine mobile robot's configuration on an arbitrary terrain profile. Nine parameters are required to fully determine the rover's configuration:

- The position of a point on the robot body. (Here we choose the rover frame reference point $\left(X_{Y}, Y_{Y}, Z_{y}\right)$ ).

- The orientation of the rover body expressed in global frame $\left(\Phi_{x^{s}} \Phi_{y,} \Phi_{z}\right)$.

- The joint angles of the rocker-bogie suspension mechanism $\left(\rho, \beta_{1}, \beta_{2}\right)$.

The transformation matrix between Global coordinate frame and contact frame is obtained using equations (10) and (11).

$T_{W, R}=\left[\begin{array}{cccc}c \Phi_{z} c \Phi_{y} & -\mathrm{s} \Phi_{\mathrm{x}} \cdot \mathrm{s} \Phi_{\mathrm{z}}+\mathrm{s} \Phi_{\mathrm{x}} \cdot \mathrm{s} \Phi_{\mathrm{y}} \cdot \mathrm{s} \Phi_{\mathrm{z}} & \mathrm{s} \Phi_{\mathrm{x}} \cdot \mathrm{s} \Phi_{\mathrm{z}}+\mathrm{c} \Phi_{\mathrm{x}} \cdot \mathrm{s} \Phi_{\mathrm{y}} \cdot \mathrm{c} \Phi_{\mathrm{z}} & X \\ s \Phi_{z} c \Phi_{y} & \mathrm{c} \Phi_{\mathrm{x}} \cdot \mathrm{c} \Phi_{\mathrm{z}}+\mathrm{s} \Phi_{\mathrm{x}} \cdot \mathrm{s} \Phi_{\mathrm{y}} \cdot \mathrm{s} \Phi_{\mathrm{z}} & -\mathrm{s} \Phi_{\mathrm{x}} \cdot \mathrm{c} \Phi_{\mathrm{z}}+\mathrm{c} \Phi_{\mathrm{x}} \cdot \mathrm{s} \Phi_{\mathrm{y}} \cdot \mathrm{s} \Phi_{\mathrm{z}} & Y \\ -s \Phi_{y} & s \Phi_{y} c \Phi_{y} & c \Phi_{y} \cdot c \Phi_{y} & Z \\ 0 & 0 & 0 & 1\end{array}\right]$

$$
T_{\mathrm{W}_{i} \mathrm{C}_{\mathrm{i}}}=\left(T_{W_{2} R}\right)\left(T_{R_{i} A_{i}}\right)\left(T_{A_{\bar{L}} C_{\mathrm{i}}}\right)
$$

Then the position and orientation of the contact coordinate frames in the global frame could be extracted:

$$
\left[\begin{array}{c}
X_{i}\left(U, q, \delta_{i}\right) \\
Y_{i}\left(U, q, \delta_{i}\right) \\
Z_{i}\left(U, q, \delta_{i}\right) \\
\Phi_{x i}\left(U, q, \delta_{i}\right) \\
\Phi_{y i}\left(U, q, \delta_{i}\right) \\
\Phi_{z i}\left(U, q, \delta_{i}\right)
\end{array}\right]=\left[\begin{array}{c}
T_{\mathrm{W}_{i} \mathrm{C}_{\mathrm{i}}}[1,4] \\
T_{\mathrm{W}_{i} \mathrm{C}_{\mathrm{i}}}[2,4] \\
T_{\mathrm{W}_{i} \mathrm{C}_{\mathrm{i}}}[3,4] \\
\tan ^{-1}\left(\frac{T \mathrm{~W}_{i} \mathrm{C}_{\mathrm{i}}[3,2]}{T \mathrm{~W}_{i} \mathrm{C}_{\mathrm{i}}[3,3]}\right) \\
\sin ^{-1}\left(-T_{\mathrm{W}_{i} \mathrm{C}_{\mathrm{i}}}[3,1]\right) \\
\tan ^{-1}\left(\frac{T \mathrm{~W}_{i} \mathrm{C}_{\mathrm{i}}[2,1]}{{ }_{\mathrm{W}_{i} \mathrm{C}_{\mathrm{i}}}[1,1]}\right)
\end{array}\right]
$$

Suppose that the terrain elevation is given by $\mathrm{Z}_{\text {ter }}(\mathrm{X}, \mathrm{Y})$. If the rover has a wheel in contact with the terrain then the elevation of the terrain at the wheel contact location must be equal to the Z-component of the contact location, thus six independent equations could be written as:

$$
Z_{\text {tey }}\left(X_{\mathrm{i} x} Y_{\mathrm{i}}\right)-Z_{\mathrm{i}}\left(X_{x} Y_{x} Z_{v} \Phi_{X^{x}} \Phi_{y^{v}} \Phi_{Z^{v}} p_{v} \beta_{1^{x}} \beta_{2}\right)=0 \quad i=1,2_{x w} 6
$$

By determining a desired path for the rover $\mathrm{X}, \mathrm{Y}, \Phi_{\mathrm{z}}$ are treated as known parameters. Then the other six parameters are found by solving (13). Contact angles could be obtained by:

$$
\delta_{i}=\tan ^{-1}\left(\Gamma_{i}\right)-\Phi_{y}-(-1)^{i+1} \rho \quad i=1,2
$$

$$
\begin{array}{lc}
\delta_{i}=\tan ^{-1}\left(\Gamma_{i}\right)-\Phi_{y}-\rho-\beta_{1} & i=3,5 \\
\delta_{i}=\tan ^{-1}\left(\Gamma_{i}\right)-\Phi_{y}+\rho-\beta_{2} & i=4,6
\end{array}
$$

Where $\Gamma_{\tilde{\mathrm{i}}}$ is the terrain slope at $\mathrm{X}_{\mathrm{i},}, \mathrm{Y}_{\mathrm{i}}$ along wheel heading $\Phi_{\text {zi }}$. The system of equations (13) is highly nonlinear. We use fsolve optimization function provided by Matlab to solve (13).

\section{Results and Discussion}

In this section we study kinematics behaviour of rover and investigate the verification of the derived model as it moves over the simulated terrain. The MD Adams Constructed prototype rover and terrain topology used for simulation is shown in fig. 6 and consists of two parts. The right side has slopes with 16.7 degrees that the right side of rover (Wheels 2,4,6) are moving on and the left side is sinusoidal hill-like terrain that wheels $1,3,5$ are moving on. The rover reference point is at position $\mathrm{X}=\mathrm{Y}=0$ at $\mathrm{t}=0.0$ and moves straight ahead with a constant velocity of $2(\mathrm{~cm} / \mathrm{sec})$ without any turning $\left(\phi_{z}=0\right)$.

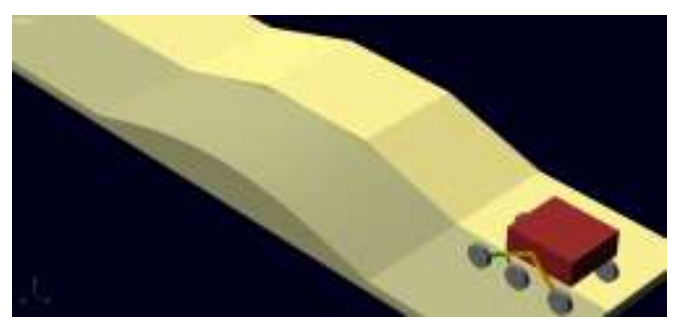

Figure 6. Terrain Profile Used for Simulation

Fig. 7 shows Rover pitch and roll angles for kinematics model and Adams simulations. The angles are in degree. The curves show good compatibility between kinematic model derived here and simulation results. We can see that as the front wheels reach the bumps both angles become negative. The pitch angle remains negative during the first half of the terrain and then turns positive as the robot crosses the top of the hill. 


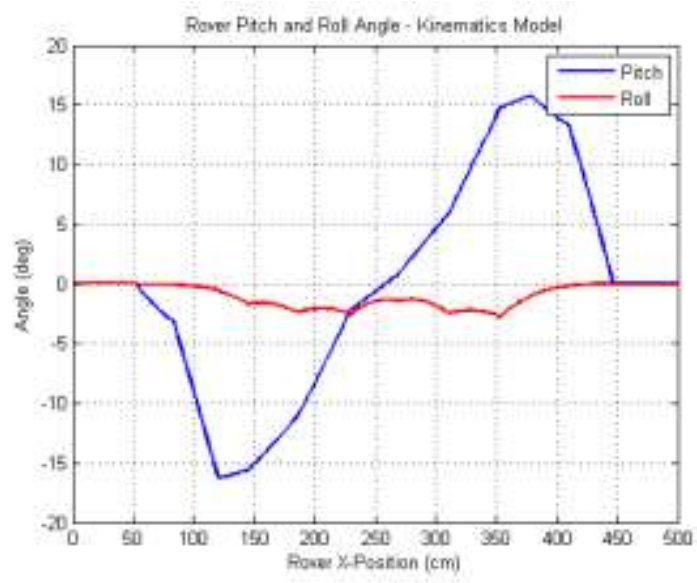

(a) Kinematics Model Results

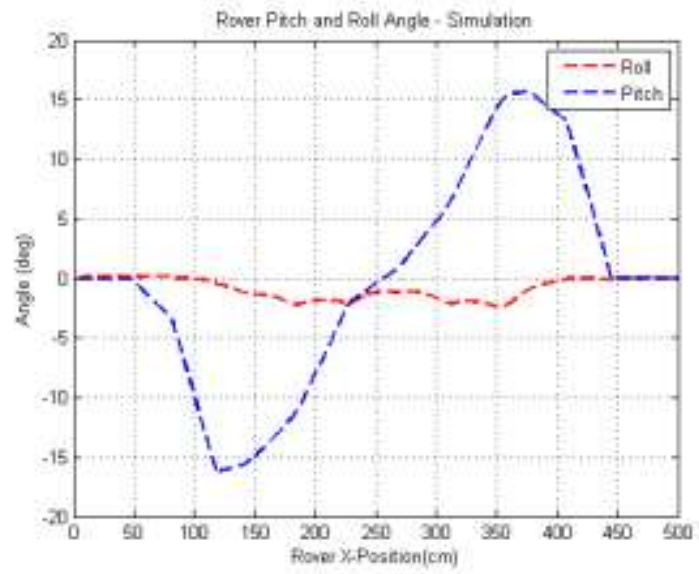

(b) Simulation Results

Figure 7. Rover Pitch and Roll

Fig. 8 shows rocker and bogie joint angles as the result of combined motion of six wheels. Again the calculated results agree with measured results. Right bogie angle is zero when all of the wheels are on the same surface but the left bogie has a harmonic curve on the hill and experiences transitions when the front and middle wheels touch the bump.

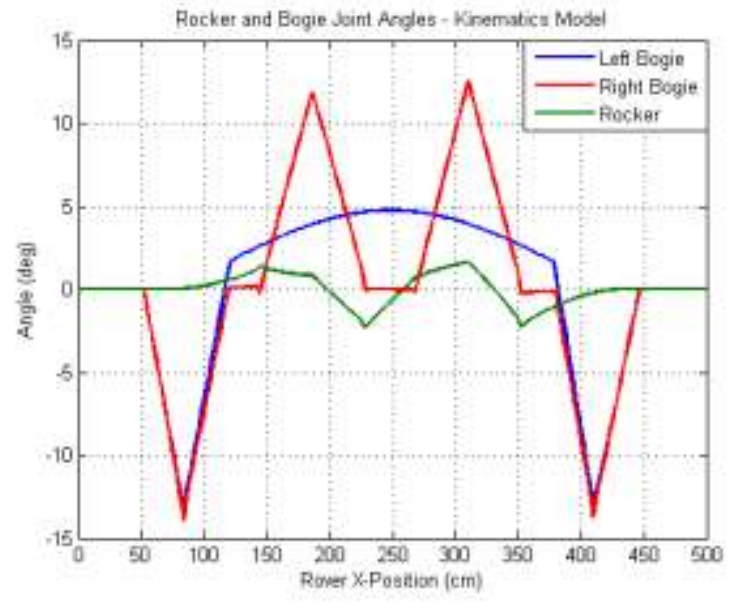

(a) Kiematics Model Results

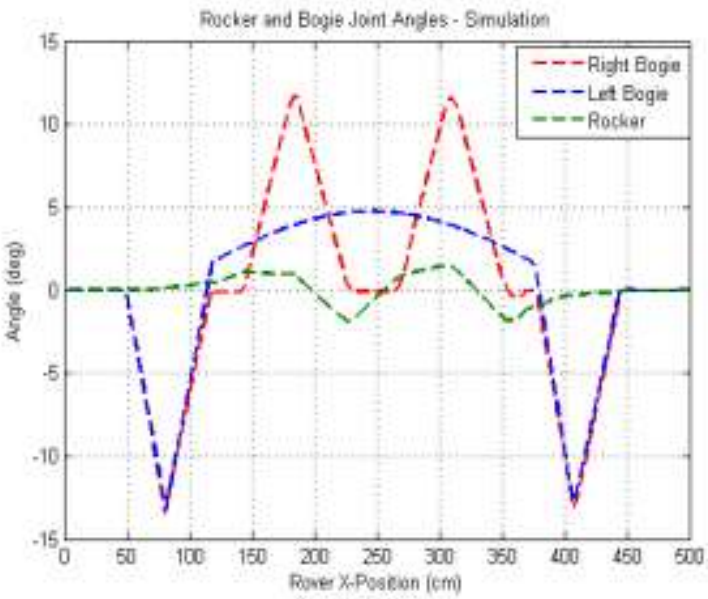

(b) Simulation Results

Figure 8. Rocker and Bogie Joint Angles

Fig. 9 shows contact angle of front wheels. Both contact angles experience an abrupt change when the wheels hit the bump. The left contact angle then trends to a positive value after the rover climbed the hill. The right contact angle is zero at all times except when the wheel is shifted between slope and flat surface. 


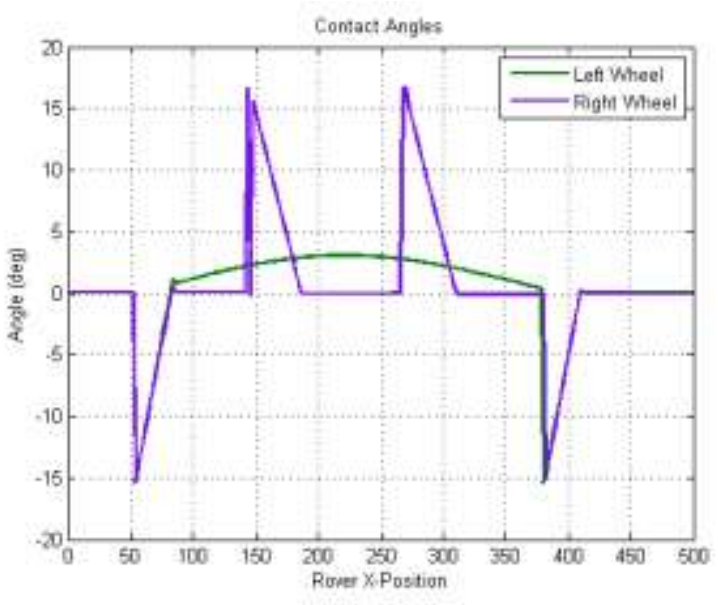

Figure 9. Contact Angle for Front Wheels

\section{Conclusion}

In this paper kinematics modelling of a mobile robot with rocker-bogie suspension system was presented and velocity equations of rover moving on uneven terrain were extracted by introducing the contact angle. By using D-H notation and instantaneous coincident coordinate concept the Jacobian matrix for each wheel was derived which relates the rover velocity vector with wheel angular velocities and joint angular rates. Then, a rover-terrain model was developed to obtain the necessary joint angles and some attitude angles by solving a set of nonlinear equations through an optimization process. To verify the kinematic model the rover was simulated by MD ADAMS and it was shown that the output of the simulation is in agree with the results of the model. Finally the limitation of the modelling is that we assume a single contact point between each wheel and a continuous smooth terrain. Also in the modelling of rover-terrain interaction some wheels may not always be in contact with the ground.

\section{References}

[1] Chottiner J.E. (1992). Simulation of a six-wheeled martian rover called the rocker-bogie. M.S. Thesis, Ohio State University, Columbus, Ohio, USA.

[2] Cox J., Wilfong G.T. (1990). Autonomous Robot Vehicles. Speringer-Verlag, N.Y.

[3] Craig J.J. (1989). Introduction to Robotics: Mechanics and Control, 2nd Edition., Addison-Wesley Pub.

[4] Hacot H. (1998). Analysis and Traction Control of a Rocker-Bogie Planetary Rover. M.S. Thesis, Massachusetts Institute of Technology. Cambridge. MA.

[5] Iagnemma K., Genot F., Dubowski S. (1999). Rapid PhysicsBased Rough Terrain Rover Planning With Sensor and Control Uncertainty. IEEE International Conference on Robotics and Automation, Detroit, MI, pp. 2286-2291.

[6] Linderman R. and Eisen H. (1992). Mobility analysis, simulation and scale model testing for the design of wheeled planetary rovers, In Missions Technologies and Design of Planetary Mobile Vehicle, Toulouse, France.

[7] Muir P.F., Neuman C.P. (1987). Kinematic modelling of wheeled mobile robots. Robotics Systems, 4(2).

[8] Tarokh M., McDermott G., Hayati S., Hung J. (1999). Kinematic modelling of a high mobility Mars rover. "IEEE Conf. on Robotics and Automation", Detroit, MI 